DOI: http://doi.org/10.52716/jprs.v11i4.560

\title{
Petroleum Hydrocarbon Quarterly Boiling Range Distribution of Iraqi Crude Oil by Simulated Distillation Method
}

\author{
Aliaa K. Alhead ${ }^{1, *}$, Shatha F. Khaleel ${ }^{2}$ \\ Ministry of Oil/ Petroleum Research and Development Center/ BaghdadL Iraq. \\ ${ }^{1, *}$ Corresponding Author E-mail: a_k_alhead @yahoo.com \\ ${ }^{2}$ E-mail: shatha_khaleel@yahoo.com
}

Received 18/2/2021, Accepted 6/6/2021, Published 20/12/2021

This work is licensed under a Creative Commons Attribution 4.0 International License.

\begin{abstract}
$\underline{\text { Abstract }}$
This study includes analysis of different crude oil stock for various field Iraqi oil by gas chromatography instrument, using simulated distillation technique for determining the initial and final boiling point distribution and specified compound distillation information (normal paraffins) (Recovery W/W) for $\left(\mathrm{nC}_{5}-\mathrm{nC}_{44}\right)$, ASTM-D5307 becomes the analytical method. This method need tow samples; the first one spiked with internal standard and the second without internal standard. This analysis for quantitative and qualitative oil characterization which is often useful for evaluating the range of hydrocarbons in crude oil using Simulated Distillation. The study was performed using:

- Quarterly analysis of SIMDIS GC Distillation for three field (East Baghdad, Badra, Amara)

- Comparison of analyzes of SIMDIS GC Distillation with Different API (light, intermediate, heavy) with Initial boiling point (IBP).

- Finding experimental relationship between API and Initial boiling point (IBP): $\mathrm{IBP}=1873.8 \mathrm{API}^{-1.28}$
\end{abstract}

The result of this study shows that the boiling point increase as the number of carbon is increase, the values of $n$-Pentane (nC5) to $n$ - Tetratetracontane (nC44) (w/w) changes from winter and summer (difference in temperatures), Positive correlation between C6 and C5 with API, where their percentages increase with increasing API for crude oil and C6 and C5 are lower in summer than in winter due to the evaporation of light components of the samples in summer. Initial boiling point increase as the API is decrease that mean in crude oil have heavy component increases and light component decrease (inverse relationship). 
توزيع معدل الغليان للهيدركريونات البترولية للنفط الخام العراقي بطريقة محاكاة التقطير

الخلاصة:

تضمن هذا البحث تحاليل للنفوط الخام العر اقية عن طريق التحليل الكمي والنوعي للنفط الخام وتقييم مجموعة الهيدروكربونات باستخدام تقنية محاكاة التقطير Simulation Distillation هي احد تقنيات كروماتوغر افيا الغاز GC وتستخدم لمر اقبة عملية التكرير لضمان المنتجات التي ينم إنتاجها كما هو منوقع.وباستخدام تقنية

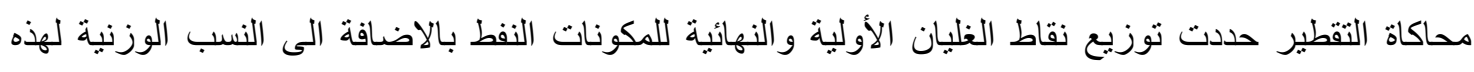
المكونات (n-C5 إلى n-C44) وباتباع الطريقة القياسية ASTM-D5307 و التي تتطلب تحليل كل عينة بحقنها مرتين؛ المرة الأولى مع المحلول القياسي (internal standard) و والثاع (المرة الثانية بدونه. تم تتفيذ البحث بالمحاور :-

- مقارنة نتائج التحليل الفصلي بتقنية محاكاة التقطير Simulation Distillation لثناثة حقول (شرق بغداد، بدرة، العمارة) و التغيير الحاصل في قيم النسب الوزنية (w/w) للمركبات ( n-C6 n-C6 )

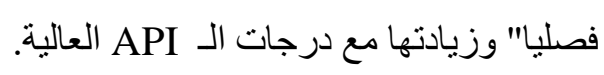

- مقارنة نتائج محاكاة التقطير مع درجات الـ API المختلفة (الخفيفة و المتوسطة و التقبلة) و نقاط الغليان الأولية (IBP) و العلاقة العكسية بينهم. - - العلاقة العملية بين API ونقطة الغليان الأولية (IBP). $\mathrm{IBP}=1873.8$ API $^{-1.28}$ بينت النتائج:

- التغير الحاصل في درجات الغليان الاولية بتغيير عدد ذرات الكاربون من ( n-C5 إلى n-C44 ) نتيجة

$$
\text { - - تغيرفي درجات الحرارة بين فصلي الثتاء و الصيف. }
$$

- التغير الواضح في النسب الوزنية للمكونات (nC5-nC44 ) بالنسبة لفصلي الثتاء و الصيف.

\section{Introduction:}

Petroleum hydrocarbons have large number of compounds found in crude oil, natural gas, coal, and peat. They consist of three major groups of compounds, the alkanes (paraffins), alkenes (olefins), and aromatics. Researchers have increased the need for sophisticated techniques in analyses for excellent hydrocarbon profiling and characterization. Gas chromatographic technique was successfully in the determination of hundreds of hydrocarbons and other organic compounds. It is an ideal tool in analyzing gas and liquid samples, thus allowing the researcher to identify both the type of molecular species present, their concentrations, and also obtain 
information from hydrocarbon samples (free product) by determining the composition of the hydrocarbons present. Crude oil varies widely in appearance and viscosity from field to field, characterization and source identification of oils from various fields is necessary. One of such ways of doing this is by analyzing the hydrocarbon fractions, Gas Chromatography analysis for quantitative and qualitative oil characterization which is often used for evaluating the range of hydrocarbons in crude oil or rock extract. [1,2]

Gas Chromatography requires analyzing entire oil for hydrocarbon range in a gas chromatograph while certain hydrocarbon ratios were employed in evaluating such things as maturity, source, biodegradation and evaporative fractionation. [3] Fingerprinting technology was developed which allows the oil a new well for each reservoir, and allows the oil from multiple wells to commingle and the respective contributions identified by source and proportion. [4]

\subsection{Petroleum Hydrocarbons Analysis:}

Crude oil sample can be analysis by injecting a small portion of the sample into a gas chromatograph. Once injected $[1]$, the product was heated and vaporized and carried into a column by a flow of inert gas. After injection the temperature of the column is slowly raised, as the temperature increases the compounds begin to move through the column, in general the more volatile and lower boiling compounds start moving first. A flame ionization detector connected to the end of the column detects the components of the product as they elute from the column. The time that it takes for individual components to go through the column depends on:

- Temperature

- Length of column

- Column characteristics

- The character of the compound

Crude oil consists of a very large number of compounds that, by defined, as stoke tank oil, as well as other sources of petroleum such as coal, and peat. Petroleum hydrocarbons consist of three major groups of compounds: 
Open Access

No. 33, December 2021, pp.1- 16

- Alkanes (paraffins)

- Alkenes (olefins),

- Aromatics.

Hydrocarbon products such as gasoline, diesel fuel, and asphalts are all derived from crude oil by a variety of refining and distillation processes. Each product is produced by the combination of multiple individual hydrocarbon compounds all of which have different vaporization and boiling temperatures. The middle boiling range compounds are used in differing proportions to create products such as kerosene, diesel, and heating oil. These products predominantly contain $\mathrm{C}_{10}$ to $\mathrm{C}_{24}$ alkanes, and polynuclear aromatics with little to no olefins. [1]

\subsection{Simulated Distillation (SD) by Gas Chromatography}

The methods (ASTM D-5307) the distribution of crude oil and determining the boiling points used Simulated Distillation is a GC technique method .This data very useful to refinery engineers in determining the process and to monitor the refining process to ensure the products produced are as expected. ASTM D5307 analytical method Simulated Distillation, Results are good agreement with the physical distillation. Simulated distillation (SimDis) is a gas chromatography (GC) technique which separates individual hydrocarbon components in the order of their boiling points. Simulated distillation is a GC method used to characterize petroleum fractions and Specified Compound Distillation information (Normal Paraffins) (Recovery W/W) for $\left(\mathrm{C}_{5}-\mathrm{C}_{44}\right)$ and their boiling range distribution. A correlation was reported between the boiling points and the percentages of the sample. [5, 6]

The methods (ASTM D-5307) of simulated distillation for crude oil and petroleum fractions by gas chromatography shown in Table (1) below, according to the device (Shimadzu Simulated Distillation Gas Chromatograph GC-2014) a suitable method is (ASTM D5307) used for this study. 
Table (1) Summary of Simulated Distillation Test Methods

\begin{tabular}{|c|c|c|c|c|}
\hline \multirow{2}{*}{ ASTM Method } & \multirow{2}{*}{$\begin{array}{l}\text { Maximum } \\
\text { Carbon \# }\end{array}$} & \multirow{2}{*}{ Sample Type } & \multicolumn{2}{|c|}{ Boiling Point Range (BP) } \\
\hline & & & Initial (IBP) & Final (FBP) \\
\hline ASTM D3710 & Up to $\mathrm{C} 15$ & $\begin{array}{l}\text { Gasoline, } \\
\text { Naphtha }\end{array}$ & $\sim-20^{\circ} \mathrm{C}$ to $30^{\circ} \mathrm{C}$ & $<260^{\circ} \mathrm{C} / 500^{\circ} \mathrm{F}$ \\
\hline ASTM D7096 & $\mathrm{C} 3$ to $\mathrm{C} 16$ & $\begin{array}{l}\text { Gasoline, } \\
\text { Naphtha }\end{array}$ & $\sim-20^{\circ} \mathrm{C}$ to $30^{\circ} \mathrm{C}$ & $<280^{\circ} \mathrm{C} / 536^{\circ} \mathrm{F}$ \\
\hline $\begin{array}{c}\text { ASTM D2887, IP } \\
\text { 404, ISO 3924, } \\
\text { DIN } 51435\end{array}$ & $\mathrm{C} 3$ to $\mathrm{C} 44$ & $\begin{array}{c}\text { Jet Fuel, Diesel, } \\
\text { Biodiesel } \\
\text { Blends }\end{array}$ & $\sim 40^{\circ} \mathrm{C}$ to $80^{\circ} \mathrm{C}$ & $<538^{\circ} \mathrm{C} / 1000^{\circ} \mathrm{F}$ \\
\hline ASTM D5442 & $\mathrm{C} 17$ to $\mathrm{C} 44$ & $\begin{array}{c}\text { Petroleum } \\
\text { derived waxes }\end{array}$ & & $<538^{\circ} \mathrm{C} / 1000^{\circ} \mathrm{F}$ \\
\hline ASTM D5307 & C3 to $\mathrm{C} 44$ & Crude Oil & $-30^{\circ} \mathrm{C}$ to $100^{\circ} \mathrm{C}$ & $<538^{\circ} \mathrm{C} / 1138^{\circ} \mathrm{F}$ \\
\hline ASTM D7398 & $\mathrm{C} 8$ to $\mathrm{C} 70$ & Biodiesel, B100 & $>100^{\circ} \mathrm{C} / 212^{\circ} \mathrm{F}$ & $<615^{\circ} \mathrm{C} / 1139^{\circ} \mathrm{F}$ \\
\hline ASTM D7213 & $\mathrm{C} 5$ to $\mathrm{C} 70$ & $\begin{array}{c}\text { Lube Oil, Base } \\
\text { Oil }\end{array}$ & $>100^{\circ} \mathrm{C} / 212^{\circ} \mathrm{F}$ & $<615^{\circ} \mathrm{C} / 1139^{\circ} \mathrm{F}$ \\
\hline ASTM D6352 & $\mathrm{C} 5$ to $\mathrm{C} 90$ & $\begin{array}{c}\text { Lube Oil, Base } \\
\text { Oil }\end{array}$ & $>100^{\circ} \mathrm{C} / 212^{\circ} \mathrm{F}$ & $<700^{\circ} \mathrm{C} / 1292^{\circ} \mathrm{F}$ \\
\hline $\begin{array}{c}\text { IP 480, EN 15199- } \\
1\end{array}$ & $\mathrm{C} 10$ to $\mathrm{C} 120$ & $\begin{array}{c}\text { Lube Oil, Base } \\
\text { Oil }\end{array}$ & $>100^{\circ} \mathrm{C} / 212^{\circ} \mathrm{F}$ & $<750^{\circ} \mathrm{C} / 1382^{\circ} \mathrm{F}$ \\
\hline ASTM D7169 & $\mathrm{C} 3$ to $\mathrm{C} 100$ & $\begin{array}{c}\text { Residues, Crude } \\
\text { Oil }\end{array}$ & $-30^{\circ} \mathrm{C}$ to $100^{\circ} \mathrm{C}$ & $<720^{\circ} \mathrm{C} / 1328^{\circ} \mathrm{F}$ \\
\hline $\begin{array}{c}\text { IP 507, IP 545, } \\
\text { EN 15199-2, } \\
\text { EN 15199-3 }\end{array}$ & Up to $\mathrm{C} 120$ & $\begin{array}{c}\text { Heavy Distillate } \\
\text { Residues, Crude } \\
\text { Oil }\end{array}$ & $>100^{\circ} \mathrm{C} / 212^{\circ} \mathrm{F}$ & $<750^{\circ} \mathrm{C} / 1382^{\circ} \mathrm{F}$ \\
\hline ASTM D7500 & $\mathrm{C} 5$ to $\mathrm{C} 110$ & $\begin{array}{c}\text { Lube Oil, Base } \\
\text { Oil }\end{array}$ & $>100^{\circ} \mathrm{C} / 212^{\circ} \mathrm{F}$ & $<735^{\circ} \mathrm{C} / 1355^{\circ} \mathrm{F}$ \\
\hline $\begin{array}{l}\text { ASTM D7900, } \\
\text { IP } 601\end{array}$ & $\mathrm{C} 1$ to $\mathrm{C} 10$ & $\begin{array}{l}\text { Stabilized } \\
\text { Crude Oil }\end{array}$ & $\sim>-30^{\circ} \mathrm{C}$ & Fraction $<170^{\circ} \mathrm{C}$ \\
\hline ASTM D6417 & $\mathrm{C} 5$ to $\mathrm{C} 22$ & $\begin{array}{l}\text { Volatility of } \\
\text { Crude Oil }\end{array}$ & $\begin{array}{c}\text { IBP to } 371^{\circ} \mathrm{C} / \\
700^{\circ} \mathrm{F}\end{array}$ & $<700^{\circ} \mathrm{C} / 1292^{\circ} \mathrm{F}$ \\
\hline
\end{tabular}




\section{Experimental Work:}

\subsection{ASTM D5307 Using the Shimadzu Simulated Distillation Gas Chromatograph}

\section{System:}

This method need to two samples was injected twice, first one with internal standard and the second one without. Two runs to determine an initial and final boiling point), ASTM D5307 test max carbon $\left(\mathrm{C}_{5}\right.$ to $\left.\mathrm{C}_{44}\right)$ and sampling type crude oil, initial boiling point (IBP) $\left(-30^{\circ} \mathrm{C}\right.$ to $\left.100^{\circ} \mathrm{C}\right)$, final boiling point $(\mathrm{FBP})\left(<538^{\circ} \mathrm{C}\right)$. Boiling point distribution graph is created for the fraction with boiling points lower than $538^{\circ} \mathrm{C}$. The fraction with boiling points above $538^{\circ} \mathrm{C}$ is calculated as a residual percentage (mass $\%)$. The Shimadzu simulated distillation gas chromatograph system, consisting of the GC-2014 and the LabSolutions distillation GC analysis software, is compliant with ASTM D5307, and combines comfortable operability with high-level functionality ,specified compound distillation information (Normal Paraffins) (Recovery W/W) for $\left(\mathrm{C}_{5}-\mathrm{C}_{44}\right)$.

\subsection{Instruments Analysis Conditions (Gas chromatograph GC-2014)}

labSolution Software included: Real time analysis program, Post run analysis program and Browser program.

\section{- Analysis Conditions:}

Column temperature injection port temperature were $\left[40{ }^{\circ} \mathrm{C}\left(10^{\circ} \mathrm{C} / \mathrm{min}\right)-350{ }^{\circ} \mathrm{C}(30\right.$ min)] and $\left(375^{\circ} \mathrm{C}\right)$ and FID temperature $\left(380^{\circ} \mathrm{C}\right)$. Carrier gas flow rate and Hydrogen flow rate were $(40 \mathrm{~mL} / \mathrm{min}$ (nitrogen $)\{40 \mathrm{~mL} / \mathrm{min}(55 \mathrm{kPa})\}$. Air flow rate $(400$ $\mathrm{mL} / \mathrm{min}(40 \mathrm{kPa}))$, Injection volume $(1 \mu \mathrm{L})$.

To calibration used two samples standard solutions were measured, one of them a mixture of $n-C_{5}$ to $n-C_{-9}$, and another mixture from $\mathrm{nC}_{10}$ to $n-C_{44}$ ) to determination of the initial and final boiling points.

This test method cover the determination of the boiling point rang distribution include:

1. SIMDIS GC Distillation Graph (is the resulting distillation curve plot between recovery $(\%)$ and the boiling point. 
2. SIMDIS GC Result Table starting at initial boiling point and listing \% distilled in $5^{\circ} \mathrm{C}$ increments up to the Final boiling point and can be defined this results as:

- Initial boiling point (IBP)-definition as the point at which a cumulative volume count first drop of the total volume count under the chromatogram is obtained.

- Final boiling point (FBP) - definition as the point at which a cumulative volume count equal to $99.5 \%$ of the total volume count under the chromatogram is obtained.

3. Specified Compound Distillation information (Normal Paraffins) (Recovery W/W) for $\left(\mathrm{C}_{5}-\mathrm{C}_{44}\right)$.

\section{Result and Discussion}

\subsection{SIMDIS GC Distillation Result:}

The crude oil samples was collected from different production Iraqi fields (North, South and Midland ) at different periods of time than stored at low temperature (in refrigerator), all samples were measured API and Shimadzu Simulated Distillation Gas Chromatograph information for the samples. The results from Instruments Gas chromatograph (GC-2014) of SIMDIS GC Distillation for samples shown in Tables (2 to 4$)$.

Table (2) Result of SIMDIS GC Distillation for South Iraqi Fields (Initial boiling point (IBP) and Final boiling point (FBP)

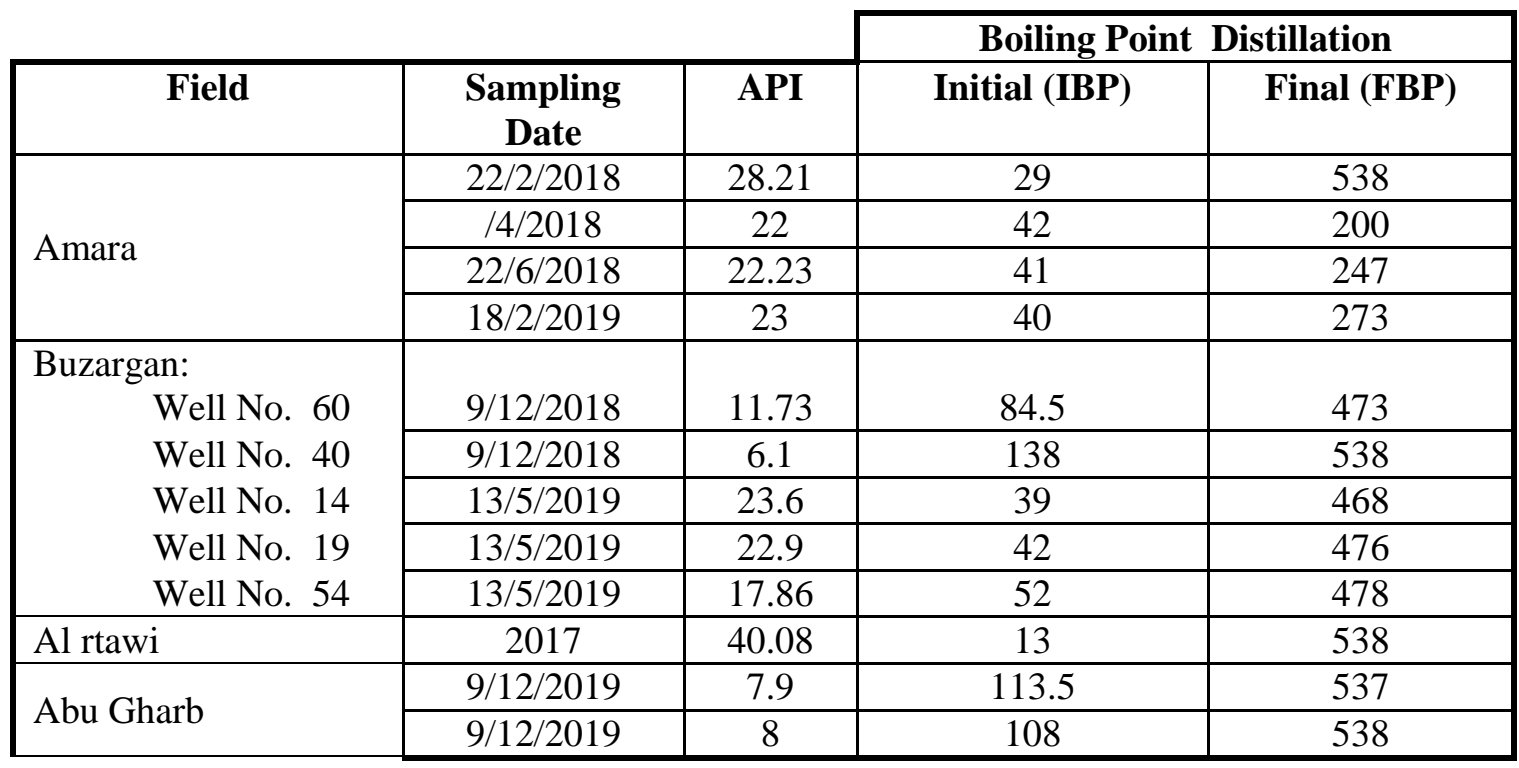


Journal of Petroleum Research and Studies

Open Access

No. 33, December 2021, pp.1- 16

P- ISSN: 2220-5381

E- ISSN: 2710-1096

\begin{tabular}{|l|c|c|c|c|}
\hline Halfaya :CPFN195A & $9 / 12 / 2018$ & 18 & 42 & 538 \\
\hline Halfaya & $26 / 7 / 2019$ & 22.51 & 36 & 480 \\
\hline Halfaya :CPF1HF7 & $9 / 12 / 2018$ & 41.44 & 12 & 537 \\
\hline Lahas & $26 / 7 / 2019$ & 31.99 & 22 & 538 \\
\hline Nahar Omar & $23 / 7 / 2019$ & 26.48 & 31 & 537 \\
\hline Majnoon & $25 / 7 / 2019$ & 32.34 & 20 & 538 \\
\hline Saba & $26 / 7 / 2019$ & 28.88 & 20 & 538 \\
\hline Tuba & $26 / 7 / 2019$ & 24.68 & 19 & 537 \\
\hline West Qurna 1 & $26 / 7 / 2019$ & 24.97 & 36 & 538 \\
\hline West Qurna 2 & $25 / 7 / 2019$ & 22.003 & 28 & 538 \\
\hline Rumila DS1 & $25 / 7 / 2019$ & 27.82 & 24 & 537 \\
\hline Rumila DS2 & $25 / 7 / 2019$ & 29.43 & 25 & 538 \\
\hline Zubair & $24 / 7 / 2019$ & 29.54 & 34 & 538 \\
\hline
\end{tabular}

Table (3) Result of SIMDIS GC Distillation for Middle Iraqi Fields

(Initial boiling point (IBP) and Final boiling point (FBP)

\begin{tabular}{|c|c|c|c|c|}
\hline & & & Boiling Poi & istillation \\
\hline Field & Sampling Date & API & Initial (IBP) & Final (FBP) \\
\hline \multirow{5}{*}{ Badra } & $2 / 3 / 2018$ & 30 & 25 & 537 \\
\hline & $/ 6 / 2018$ & 33.5 & 28 & 446.5 \\
\hline & $1 / 11 / 2018$ & 34.11 & 34.1 & 356 \\
\hline & $23-4-2019$ & 33.9 & 34.5 & 352 \\
\hline & $1 / 8 / 2019$ & 35.03 & 27 & 520 \\
\hline \multirow[t]{4}{*}{ East Baghdad } & $6 / 2018$ & 22.06 & 33 & 537 \\
\hline & $16 / 10 / 2018$ & 20.16 & 36.5 & 537 \\
\hline & $2 / 4 / 2019$ & 21.73 & 30 & 538 \\
\hline & $8 / 7 / 2019$ & 22.17 & 48 & 538 \\
\hline \multirow[t]{2}{*}{ Neft Khana } & $9 / 12 / 2018$ & 40.88 & 13 & 536 \\
\hline & $8 / 7 / 2019$ & 40.96 & 32 & 537 \\
\hline \multirow[t]{2}{*}{ Ahadab } & $27 / 3 / 2018$ & 22.5 & 30 & 334.5 \\
\hline & $1 / 8 / 2019$ & 25.29 & 41 & 363 \\
\hline
\end{tabular}


Table (4) Result of SIMDIS GC Distillation for North Iraqi Fields (Initial boiling point (IBP) and Final boiling point (FBP)

\begin{tabular}{|c|c|c|c|c|}
\hline & & & Boiling $\mathbf{P}$ & istillation \\
\hline Field & $\begin{array}{c}\text { Sampling } \\
\text { Date }\end{array}$ & API & Initial (IBP) & Final (FBP) \\
\hline \multirow{2}{*}{ Khabaz } & $6 / 2018$ & 35 & 12 & 420.5 \\
\hline & $9 / 12 / 2018$ & 33.7 & 13 & 525 \\
\hline Bai-Hassan (South) & $9 / 12 / 2018$ & 25.64 & 27 & 537.9 \\
\hline Bai- Hassan (North) & $9 / 12 / 2018$ & 26.9 & 34.5 & 486.5 \\
\hline Jambour( North) & $9 / 12 / 2018$ & 36.53 & 15 & 537.3 \\
\hline \multirow[t]{2}{*}{ Qiaruh } & $18 / 6 / 2019$ & 15.4 & 31 & 538 \\
\hline & & 16 & 39 & 538 \\
\hline
\end{tabular}

\subsection{Comparison of Analyzes of SIMDIS GC Distillation with Different API (light,} Intermediate, Heavy) with Initial Boiling Point (IBP)

There are three types of crude oil according to API gravity (light, intermediate, heavy) [7], Table (5) below show the range of API crude oil with initial boiling point (result from SIMDIS GC Distillation).

Result of initial boiling point from SIMDIS Distillation with different API in Figure (1) shown at high API low initial boiling point that mean inverse relationship, initial boiling point increase as the API is decrease that mean in crude oil have heavy component increase and light component decrease (inverse relationship). Table (6) shows the Specified Compound Distillation Information Recovery (W/W) (Normal Paraffins) for Different API that there is a positive correlation between C6 and C5 with API, where their percentages increase with increasing API for crude oil.

Table (5) Crude Type, Based on API gravity and Initial Boiling Point (IBP)

\begin{tabular}{|c|c|c|c|}
\hline Crude Type & API Gravity & API Range & Initial Boiling Point (IBP) Range ${ }^{\circ} \mathrm{C}$ \\
\hline Light & $>30$ & $30-41$ & $\leq 15$ \\
\hline Intermediate & $20-30$ & $20.16-30$ & $>35$ \\
\hline Heavy & $<20$ & $6.1-20.16$ & $138-30$ \\
\hline
\end{tabular}


Open Access

No. 33, December 2021, pp.1- 16

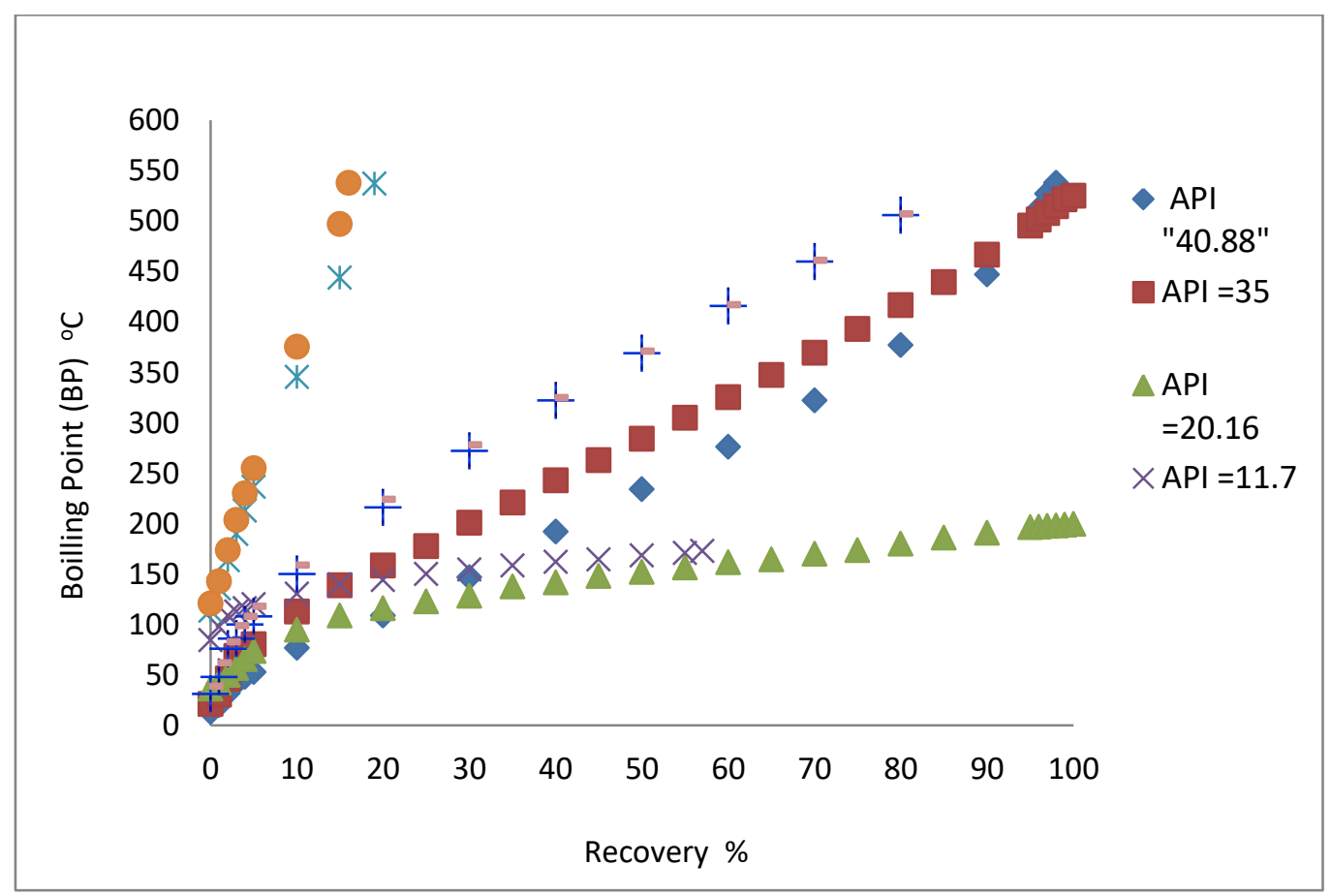

Fig. (1) SIMDIS Distillation Graf with Different API

Table (6) Specified Compound Distillation Information Recovery (W/W) (Normal Paraffins) for Different API

\begin{tabular}{|c|c|c|c|c|c|c|}
\hline API & 40.88 & 35 & 20.16 & 11.73 & 7.9 & 6.1 \\
\hline $\begin{array}{l}\text { Initial Boiling } \\
\text { Point(IPB) }\end{array}$ & 16 & 18 & 36.5 & 84.5 & 112 & 183 \\
\hline \multicolumn{7}{|c|}{ Specified Compound Distillation Information Recovery ( Normal Paraffins ) (W/W) } \\
\hline n- Pentane $-C_{5}$ & 7.6 & 5.7 & 1.4 & 1.0 & 1 & 0.6 \\
\hline n- Hexane $-\mathrm{C}_{6}$ & 5.3 & 2.8 & 1.1 & 1.0 & 0 & 0.9 \\
\hline $\mathrm{n}$ - heptane $-\mathrm{C}_{7}$ & 7.9 & 5 & 3.3 & 3.1 & 0.7 & 3 \\
\hline n- Octane $-C_{8}$ & 6.7 & 5.5 & 4.2 & 3.5 & 2.3 & 3.8 \\
\hline n-Nonane $-\mathrm{C}_{9}$ & 5.5 & 4.9 & 3.7 & 3.3 & 3 & 3.9 \\
\hline n-Deecane $-\mathrm{C}_{10}$ & 4.9 & 4.6 & 3.8 & 3.3 & 3.8 & 4.1 \\
\hline n- Undecane $-C_{11}$ & 5.2 & 4.7 & 3.5 & 3.2 & 3.7 & 4 \\
\hline n- Dodecane $-C_{12}$ & 5 & 4.3 & 3.3 & 3.1 & 3.7 & 4 \\
\hline n- Tridecane $-C_{13}$ & 4.6 & 4 & 3.2 & 2.9 & 3.6 & 3.7 \\
\hline n- Tetradecane $-C_{14}$ & 3.9 & 3.7 & 3.2 & 3 & 3.7 & 3.9 \\
\hline n- Pentadecane $-C_{15}$ & 4 & 3.7 & 3.2 & 2.9 & 3.7 & 3.8 \\
\hline n- Hexadecane $-\mathrm{C}_{16}$ & 3.5 & 3.3 & 2.9 & 2.7 & 3.4 & 3.4 \\
\hline n- Heptadecane $-C_{17}$ & 3.2 & 3.1 & 2.8 & 2.6 & 3.3 & 3.3 \\
\hline$\left(\mathrm{C}_{18}-\mathrm{C}_{19}\right)$ & 4.4 & 4.5 & 4.1 & 3.8 & 4.7 & 4.7 \\
\hline$\left(\mathrm{C}_{20} \mathrm{C}_{23}\right)$ & 6.7 & 7.3 & 7.3 & 6.8 & 8.4 & 8.4 \\
\hline$\left(\mathrm{C}_{24}-\mathrm{C}_{27}\right)$ & 7.1 & 8.4 & 8.6 & 7.8 & 9.8 & 9.3 \\
\hline$\left(\mathrm{C}_{28}-\mathrm{C}_{31}\right)$ & 4.8 & 6.5 & 7.8 & 7.4 & 9 & 8.1 \\
\hline
\end{tabular}




\begin{tabular}{|c|c|c|c|c|c|c|}
\hline$\left(\mathrm{C}_{32}-\mathrm{C}_{35}\right)$ & 3.3 & 4.9 & 6.7 & 6.7 & 7.2 & 6.5 \\
\hline$\left(\mathrm{C}_{36}-\mathrm{C}_{39}\right)$ & 2.3 & 3.7 & 5.5 & 6.4 & 5.7 & 5.3 \\
\hline$\left(\mathrm{C}_{40}-\mathrm{C}_{43}\right)$ & 1.6 & 2.9 & 4.6 & 6.1 & 4.6 & 4.3 \\
\hline n- Tetratetracontane $-\mathrm{C}_{44}$ & 2.3 & 6.5 & 15.8 & 19.4 & 14.7 & 11 \\
\hline Total & 100 & 100 & 100 & 100 & 100 & 100 \\
\hline
\end{tabular}

\subsection{Relationship between API and Initial Boiling Point}

Finding experimental relationship between API and Initial boiling point (IBP) Plot of experiment results of API and initial boiling point Figure (2) to obtain experimental equation according to best-fit line are:

$$
\begin{aligned}
& \mathrm{IBP}=1873.8 \text { API }^{-1.28} \\
& \mathrm{R}^{2}=0.93
\end{aligned}
$$

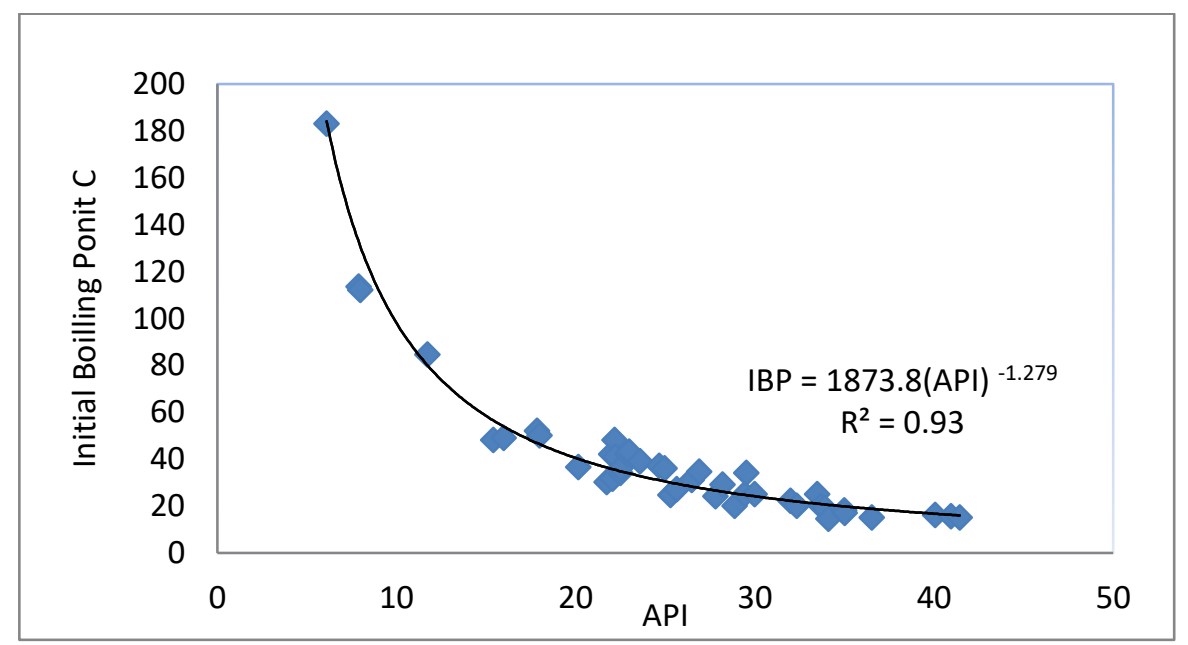

Fig. (2) Relationship between Initial Boiling Point and API

\subsection{Quarterly Analysis for Three Iraqi Fields (East Baghdad, Badra, Amara)}

Tables (7, 8 and 9) Showed different samples were took in different time (Winter, Summer) and analysis SIMDIS GC Distillation \{Specified Compound Distillation Information Recovery (Normal Paraffins) (W/W) \} and API at each sampling date for three Iraqi fields (East Baghdad, Badra, and Amara) by the Shimadzu Simulated Distillation Gas Chromatograph System (using ASTM D5307).

The results of the tables below show that: 
- Boiling point increase as the number of carbon is increase when the temperature increases.

- The values of n-Pentane (nC5) and n- Tetratetracontane (nC44) (w/w) changes in winter and summer.

- Positive correlation between C6 and C5 with API, where their percentages increase with increasing API for crude oil.

- nC6 and nC5 are lower in summer than in winter due to the evaporation of light components of the samples in summer (change in temperature).

\section{Table (7) Specified Compound Distillation Information Recovery (Normal} Paraffins) for Quarterly Analysis for East Baghdad Iraqi Field

\begin{tabular}{|c|c|c|c|c|}
\hline Field & \multicolumn{4}{|c|}{ East Baghdad } \\
\hline Sampling Date & $2-2018$ & $16-10-2018$ & $2-4-2019$ & $8-7-2019$ \\
\hline API & 22.06 & 20.16 & 21.73 & 22.17 \\
\hline $\begin{array}{l}\text { Initial Boiling Point } \\
\text { (IPB) }\end{array}$ & 33 & 36.5 & 30 & 48 \\
\hline & \multicolumn{4}{|c|}{$\begin{array}{l}\text { Specified Compound Distillation Information Recovery } \\
\text { (Normal Paraffins) (W/W) }\end{array}$} \\
\hline n- Pentane $-C_{5}$ & 1.6 & 1.4 & 1.6 & 1.7 \\
\hline n- Hexane $-\mathrm{C}_{6}$ & 1 & 1.1 & 1.3 & 1.4 \\
\hline $\mathrm{n}$ - heptane $-\mathrm{C}_{7}$ & 3 & 3.3 & 3.8 & 3.8 \\
\hline n- Octane $-C_{8}$ & 3.8 & 4.2 & 4.7 & 4.3 \\
\hline n-Nonane $-\mathrm{C}_{9}$ & 3.4 & 3.7 & 4 & 3.8 \\
\hline n-Deecane $-C_{10}$ & 3.7 & 3.8 & 4 & 3.8 \\
\hline n- Undecane $-\mathrm{C}_{11}$ & 3.4 & 3.5 & 3.7 & 3.5 \\
\hline n- Dodecane $-C_{12}$ & 3.3 & 3.3 & 3.5 & 3.6 \\
\hline n- Tridecane $-C_{13}$ & 3.2 & 3.2 & 3.3 & 3.4 \\
\hline n- Tetradecane $-C_{14}$ & 3.3 & 3.2 & 3.3 & 3.3 \\
\hline n- Pentadecane $-C_{15}$ & 3.2 & 3.2 & 3.2 & 3.2 \\
\hline n- Hexadecane $-\mathrm{C}_{16}$ & 3.9 & 2.9 & 2.9 & 2.9 \\
\hline n- Heptadecane $-C_{17}$ & 2.8 & 2.8 & 2.8 & 2.9 \\
\hline$\left(\mathrm{C}_{18}-\mathrm{C}_{19}\right)$ & 4.2 & 4.1 & 4.1 & 4.2 \\
\hline$\left(\mathrm{C}_{20-} \mathrm{C}_{23}\right)$ & 7.5 & 7.3 & 7.2 & 7.5 \\
\hline$\left(\mathrm{C}_{24}-\mathrm{C}_{27}\right)$ & 8.6 & 8.6 & 8.3 & 8.6 \\
\hline$\left(\mathrm{C}_{28}-\mathrm{C}_{31}\right)$ & 7.8 & 7.8 & 7.5 & 7.8 \\
\hline$\left(\mathrm{C}_{32}-\mathrm{C}_{35}\right)$ & 6.7 & 6.7 & 6.7 & 6.5 \\
\hline$\left(\mathrm{C}_{36}-\mathrm{C}_{39}\right)$ & 5.6 & 5.5 & 5.2 & 5.4 \\
\hline$\left(\mathrm{C}_{40}-\mathrm{C}_{43}\right)$ & 6.6 & 4.6 & 4.3 & 4.6 \\
\hline n- Tetratetracontane- $\mathrm{C}_{44}$ & 13.4 & 15.8 & 14.6 & 13.8 \\
\hline Total & 100 & 100 & 100 & 100 \\
\hline
\end{tabular}


Table (8) Specified Compound Distillation Information Recovery (Normal Paraffins) for Quarterly analysis for Amara Iraqi field

\begin{tabular}{|c|c|c|c|}
\hline Field & \multicolumn{3}{|c|}{ Amara } \\
\hline Sampling Date & $22 / 2 / 2018$ & $4 / 2018$ & $18 / 2 / 2019$ \\
\hline API & 28 & 22 & 23 \\
\hline Initial Boiling Point(IPB) & 29 & 42 & 40 \\
\hline \multicolumn{4}{|c|}{ Specified Compound Distillation Information Recovery ( Normal Paraffins ) (W/W) } \\
\hline n- Pentane $-\mathrm{C}_{5}$ & 2.2 & 1.5 & 1.1 \\
\hline n- Hexane $-\mathrm{C}_{6}$ & 1.7 & 1.3 & 1.4 \\
\hline $\mathrm{n}$ - heptane $-\mathrm{C}_{7}$ & 4.8 & 4.3 & 3.6 \\
\hline n- Octane $-\mathrm{C}_{8}$ & 5.2 & 5.3 & 4.9 \\
\hline n-Nonane $-\mathrm{C}_{9}$ & 4.7 & 4.5 & 4.2 \\
\hline n-Deecane $-\mathrm{C}_{10}$ & 4.6 & 4.6 & 4.1 \\
\hline n- Undecane $-C_{11}$ & 4.2 & 4 & 4.3 \\
\hline n- Dodecane $-C_{12}$ & 4.1 & 3.7 & 4.2 \\
\hline n- Tridecane $-C_{13}$ & 3.8 & 3.6 & 4 \\
\hline n- Tetradecane $-C_{14}$ & 3.8 & 3.7 & 3.7 \\
\hline n- Pentadecane $-C_{15}$ & 3.6 & 3.6 & 3.6 \\
\hline n- Hexadecane $-\mathrm{C}_{16}$ & 3.3 & 3.3 & 3.4 \\
\hline n- Heptadecane $-\mathrm{C}_{17}$ & 3.1 & 3.1 & 3.3 \\
\hline$\left(\mathrm{C}_{18}-\mathrm{C}_{19}\right)$ & 4.7 & 4.4 & 4.8 \\
\hline$\left(\mathrm{C}_{20-} \mathrm{C}_{23}\right)$ & 7.8 & 8 & 7.8 \\
\hline$\left(\mathrm{C}_{24}-\mathrm{C}_{27}\right)$ & 8.5 & 8.9 & 9.3 \\
\hline$\left(\mathrm{C}_{28}-\mathrm{C}_{31}\right)$ & 7.2 & 8.1 & 7.6 \\
\hline$\left(\mathrm{C}_{32}-\mathrm{C}_{35}\right)$ & 5.7 & 7.3 & 5.9 \\
\hline$\left(\mathrm{C}_{36}-\mathrm{C}_{39}\right)$ & 4.5 & 7.1 & 4.6 \\
\hline$\left(\mathrm{C}_{40}-\mathrm{C}_{43}\right)$ & 3.6 & 2.3 & 3.6 \\
\hline n- Tetratetracontane $-C_{44}$ & 8.9 & 7.4 & 11.1 \\
\hline Total & 100 & 100 & 100 \\
\hline
\end{tabular}


Open Access

No. 33, December 2021, pp.1- 16

P- ISSN: 2220-5381

E- ISSN: 2710-1096

Table (9) Specified Compound Distillation Information Recovery (Normal Paraffins) for Quarterly Analysis for Badra Iraqi Field

\begin{tabular}{|c|c|c|c|c|c|}
\hline Field & \multicolumn{5}{|c|}{ Badra } \\
\hline Sampling Date & $2 / 3 / 2018$ & $5 / 2018$ & $1 / 11 / 2018$ & $23 / 4 / 2019$ & $8-7-2019$ \\
\hline API & 30 & 33.5 & 34.11 & 33.9 & 35.03 \\
\hline $\begin{array}{l}\text { Initial Boiling } \\
\text { Point(IPB) }\end{array}$ & 25 & 28 & $34-33$ & 34.5 & 17 \\
\hline & \multicolumn{5}{|c|}{$\begin{array}{c}\text { Specified Compound Distillation Information Recovery } \\
\text { (Normal Paraffins ) (W/W) }\end{array}$} \\
\hline n- Pentane $-\mathrm{C}_{5}$ & 2 & 1.9 & 1.3 & 1.8 & 1.7 \\
\hline n- Hexane $-\mathrm{C}_{6}$ & 1.7 & 1.4 & 1.8 & 1.5 & 1.8 \\
\hline $\mathrm{n}$ - heptane $-\mathrm{C}_{7}$ & 4.9 & 4.1 & 4.8 & 4.7 & 5.1 \\
\hline n- Octane $-\mathrm{C}_{8}$ & 5.8 & 5.2 & 5.8 & 5.6 & 5.6 \\
\hline n-Nonane $-\mathrm{C}_{9}$ & 4.8 & 4.7 & 5 & 5.0 & 5 \\
\hline n-Deecane $-C_{10}$ & 5 & 5 & 5 & 5.0 & 4.9 \\
\hline n- Undecane $-C_{11}$ & 4.3 & 4.3 & 3.4 & 4.4 & 4.3 \\
\hline n- Dodecane $-C_{12}$ & 3.9 & 3.9 & 3.9 & 4 & 4.1 \\
\hline n- Tridecane $-C_{13}$ & 3.7 & 3.7 & 3.7 & 3.8 & 3.8 \\
\hline n- Tetradecane $-\mathrm{C}_{14}$ & 3.7 & 3.7 & 3.7 & 3.7 & 3.8 \\
\hline n- Pentadecane $-C_{15}$ & 3.5 & 3.5 & 3.5 & 3.5 & 3.6 \\
\hline n- Hexadecane $-\mathrm{C}_{16}$ & 3 & 3.1 & 3.1 & 3.2 & 3.2 \\
\hline n- Heptadecane $-\mathrm{C}_{17}$ & 2.9 & 3.0 & 2.9 & 3.1 & 3.1 \\
\hline$\left(\mathrm{C}_{18}-\mathrm{C}_{19}\right)$ & 4.2 & 4.3 & 4.2 & 4.2 & 4.5 \\
\hline$\left(\mathrm{C}_{20-} \mathrm{C}_{23}\right)$ & 7 & 7.2 & 7.2 & 7.4 & 7.5 \\
\hline$\left(\mathrm{C}_{24}-\mathrm{C}_{27}\right)$ & 7.6 & 7.9 & 7.8 & 7.7 & 8.1 \\
\hline$\left(\mathrm{C}_{28}-\mathrm{C}_{31}\right)$ & 6.4 & 6.7 & 6.6 & 7.6 & 6.8 \\
\hline$\left(\mathrm{C}_{32}-\mathrm{C}_{35}\right)$ & 5.1 & 5.3 & 5.2 & 5.4 & 5.4 \\
\hline$\left(\mathrm{C}_{36}-\mathrm{C}_{39}\right)$ & 4.1 & 4.2 & 4 & 4 & 4.3 \\
\hline$\left(\mathrm{C}_{40}-\mathrm{C}_{43}\right)$ & 3.2 & 3.5 & 3.2 & 3.3 & 3.4 \\
\hline n-Tetratetracontane- $C_{44}$ & 13.2 & 13.4 & 13.9 & 11.1 & 10 \\
\hline Total & 100 & 100 & 100 & 100 & 100 \\
\hline
\end{tabular}




\section{Conclusion:}

- Petroleum Hydrocarbons Analysis by gas chromatograph using SIMDIS GC Result is valuable in planning the development of the fields.

- Specified Compound Distillation Recovery (W/W) from Hydrocarbon Distillation accumulated assist to prediction of the type of components will be change (like heavy hydrocarbons).

- Changing in the result starting at initial boiling point(IBP) up to the Final boiling point(FBP) and, Specified compound distillation information (Normal Paraffins ) (Recovery W/W) for $\left(\mathrm{nC}_{5}-\mathrm{n} \mathrm{C}_{44}\right)$ between Winter and Summer.

- $\quad$ Boiling point increase as the number of carbon is increase.

- The values of $n$ - Pentane -C5 and n- Tetratetracontane -C44 (w/w) changes from Winter and Summer for example C6 and C5 are lower in Summer than in winter due to the evaporation of light components of the samples in summer.

- Positive correlation between C6 and C5 with API, where their percentages increase with increasing API for crude oil and the percentages.

- Inverse experimental relationship between API and initial boiling point (IBP). 


\section{Reference}

1. John W. Wigger, Bruce E. Torkelson, " Petroleum Hydrocarbon Fingerprinting - NumericalInterpretation Developments", P.E. Environmental Liability Management, Torkelson Geochemistry. Inc., Tulsa, Oklahoma.

2. Hunt, J.M., "Petroleum Geochemistry and Geology", Freeman and company, New York, USA p. 231 - 238, 1996.

3. www.scholarsresearchlibrary.com, http://scholarsresearchlibrary.com/archive.ht ml Scholars Research Library Archives of Applied Science Research, 2012, 4 (1):246-253.

4. Leag Oil .com Information, Paper No.2, December 2003.

5. Dan C. Villalanti, Joseph C. Raia, and Jim B. Maynard, " High-temperature Simulated Distillation Applications in Petroleum Characterization", Encyclopedia of Analytical Chemistry, R.A. Meyers (Ed.), pp. 6726-6741, John Wiley \& Sons Ltd, Chichester, 2000.

6. Mark W. Frye and William E. Harriso, "Petroleum Hydrocarbon Fingerprinting Quantitative Interpretation: Development and Case Study for Use in Environmental Forensic Investigations”, Environmental Geosciences, 1999. 